\title{
EPIDEMIOLOGY AND TREATMENT OF FUNGAL INFECTIONS IN ONCO-PEDIATRIC PATIENTS AT A REFERRAL HOSPITAL
}

\author{
Jéssica Neves Bitencourt ${ }^{1}$ \\ Lillian Morais Silva ${ }^{2}$ \\ Renata de Bastos Ascenço Soares ${ }^{3}$ \\ Cássia Silva de Miranda Godoy ${ }^{4}$
}

\begin{abstract}
Aim: to identify the epidemiology of fungal infections in the pediatric service of a reference hospital for the treatment of cancer for 2016 to 2019, through the dispensing of antifungal drugs by the hospital pharmacy. Methods: Retrospective cohort analysis of 1.211 antifungal requests. Results and Discussion: 1.211 treatments with antifungal agents were performed in the period, with $71.9 \%$ of cases treated with empirical therapy, $64.1 \%$ of cases of mucocutaneous candidiasis and with the use of nystatin in $48.6 \%$ of cases. There were 114 episodes of IFIs, in which probable fungal pneumonia represented $40.4 \%$ of the therapeutic indications, based on suggestive tomographic images and clinical and epidemiological criteria in $40.3 \%$ of cases. Conclusions: There was an agreement between the epidemiology of fungal infections and the worldwide epidemiology, but there was disagreement between the therapies applied in the study hospital and the recent guidelines.
\end{abstract}

Key words: Fungal infections. Pediatrics. Cancer. Therapy.

\footnotetext{
${ }^{1}$ Graduanda em Medicina Pontifícia Universidade Católica de Goiás (PUC Goiás) Goiânia GO - Brasil. jsknevesb@gmail.com. (D) https://orcid.org/0000-0001-5241-5505.

${ }^{2}$ Graduanda em Medicina Pontifícia Universidade Católica de Goiás (PUC Goiás) Goiânia GO - Brasil. moraisliams@gmail.com. (D) https://orcid.org/0000-0001-5643-4594.

${ }^{3}$ Professora Adjunta do Curso de Medicina, Escola de Ciências Médicas e da Vida, Pontifícia Universidade Católica de Goiás (PUC Goiás) Goiânia/GO - Brasil. $\bowtie$ renatasuarezbastos@gmail.com. https://orcid.org/0000-0003-1029-325X.

${ }^{4}$ Professora Assistente do Curso de Medicina, Escola de Ciências Médicas e da Vida, Pontifícia Universidade Católica de Goiás (PUC Goiás) Goiânia/GO - Brasil. Chefe do Controle de Infecção Hospitalar, Hospital Araújo Jorge, Associação de Combate ao Câncer em Goiás $₫$ cassiamirandagodoy@hotmail.com. https://orcid.org/0000-0003-4893-0541.
}

Recebido em Aceito em Publicado em

13/07/2021 03/09/2021




\section{INTRODUCTION}

Pediatric neoplastic disease is linked to inherited genetic factors or acquired mutations, and is therefore not a preventable disease. Data provided by the National Cancer Institute (INCA) estimate that, for each year of the 2020-2022 triennium, Brazil will have an incidence of 138,44 cancers per 1 million children and adolescents (0-19 years), totaling about 8.460 cases per year. ${ }^{1}$ In the population in question, the most common types of cancer are: leukemias, central nervous system tumors and lymphomas. ${ }^{2}$

In the population of pediatric cancer patients, there is an increased risk of opportunistic infectious diseases, such as invasive fungal infection (IFI), defined as one that occurs mainly in immunocompromised and severely ill children. IFI is related to high morbidity and mortality, despite the availability of new antifungals. Other risk groups for IFI are: premature newborns, especially those weighing less than $1.5 \mathrm{~kg}$; children undergoing treatment with bone marrow transplantation; long-term children in intensive care units (ICU); children with immunodeficiencies, such as those with HIV; or under treatment with immunosuppressants. $^{3}$

In recent years, IFI cases have increased due to clinical situations that cause severe and/or long-term neutropenia such as: treatment of hematological cancers; bone marrow and solid organ transplants; the occurrence of graft versus host disease (GVHD); corticotherapy; and the extensive use of immunosuppressants. In oncohematological patients, especially in children, the most common pathogenic fungi stand out, such as species of the genus Candida spp. and Aspergillus spp., verifying the emergence of cases diagnosed by species of "Candida non-albicans" and "Aspergillus non-fumigatus" due to the improvement in diagnostic tools and generalization of antifungal therapy. ${ }^{4}$

The clinical presentation of IFIs can be nonspecific, atypical or insidious, which makes the diagnostic approach even more challenging. In addition, the amount of opportunistic fungi has been growing, and the etiological diagnosis, knowledge of regional epidemiology and patterns of sensitivity to antifungal agents are essential for therapeutic success. $^{5}$

In 2002, the Mycoses Study Group (MSG), along with other study groups, defined the IFIs according to three levels of probability for diagnosis in immunocompromised cancer patients, namely: "proven", which needs the detection of the fungus by histological analysis 
or culture of a clinical specimen, such as fluid or biopsy, from the infectious focus; "probable" or "possible", where three elements are used: host factors that indicate it as a risk group; clinical signs and symptoms consistent with fungal infection; and mycological evidence using culture and microscopic analysis as diagnostic tests (eg galactomannan antigen search for Aspergillus spp). ${ }^{6}$

The diagnosis of fungal infection made by detecting the fungus through culture and microscopy is considered the gold standard for diagnosis. In addition, other complementary diagnostic methods can be used, such as: radiological imaging studies and detection of fungal components (antigens, RT-PCR) in tissues, body fluids or blood. Studies demonstrate that cell wall polysaccharides of some fungi can be serum biomarkers of IFIs, highlighting methods of detection of galactomannan and $\beta$-d-glucan. ${ }^{7}$

Studies show that the main antifungal agents used in oncohematological patients or those who have undergone organ transplantation are: fluconazole, itraconazole, voriconazole, posaconazole, caspofungin, micafungin, anidulafungin and amphotericin B and their formulations lipids. ${ }^{3}$

Different antifungal use strategies have been employed to reduce the negative impact on the prognosis of IFIs, including antifungal prophylaxis in high-risk hematologic patients, empirical antifungal therapy, preemptive therapy and the actual treatment of proven fungal infections. Regarding prophylaxis, it can be divided into two classes: primary and secondary. Primary prophylaxis should be indicated in patients at high risk for fungal infection who do not yet present clinical manifestations at the time of indication. In turn, secondary prophylaxis will be indicated after antifungal therapy in order to prevent the relapse of IFIs during a new period of risk. ${ }^{8}$ Empirical antifungal therapy consists of the early use of a systemic antifungal agent in neutropenic patients with persistent fever (afterwards 4 to 6 days) or recurrent despite the use of a broad-spectrum antibacterial regimen, which has no defined etiology. ${ }^{7}$ The targeted therapy itself will be used in patients with diagnostic confirmation of fungal infection based on the identification of the pathogen and the clinical condition and previous therapeutic history of the patient, however, it is a little used therapy, since the diagnosis of IFI in pediatric patients is complex due to the lack of specific radiological signs, low specificity of laboratory tests and limitations in the indication of invasive procedures. ${ }^{8}$ 
The morbidity of immunocompromised patients with IFI is high. Studies indicate that, in patients with IFI by Candida spp, the lethality rate varies between $20 \%$ and $40 \%$, while in patients with infection by Aspergillus spp. have the aforementioned rate between $50 \%$ and $90 \%$, with the highest lethality in patients with disseminated disease and/or involvement of the central nervous system. ${ }^{8}$

Given the difficulties presented in the diagnosis of invasive fungal infections in pediatric oncohematological patients and their high morbidity and mortality, this study seeks to understand the epidemiology of fungal infections in pediatric patients, the diagnostic criteria used, the consumption of antifungal drugs and the evolution of infections invasive fungal diseases admitted to a state reference institution.

The general objective was to identify the epidemiology of fungal infections in the pediatric service of a reference hospital for the treatment of cancer in the state of Goias, from 2016 to 2019, through the dispensing of antifungal drugs by the hospital pharmacy. The specific objectives were: to define the epidemiological profile of pediatric patients at risk for invasive fungal infections, according to age group, gender, underlying disease, number of hospitalizations, medications used and other comorbidities; analyze the use of drugs involved in the treatment and prevention of fungal infections in pediatric patients undergoing cancer treatment; evaluate the justification for the use of antifungal agents, the diagnosis of fungal infection and the evolution of patients undergoing antifungal therapy; and provide data and information necessary for early diagnosis and adequate management for the treatment and better prognosis of fungal infections.

\section{METHODS}

Ethical issues: the study complied with the resolutions of the National Health Council (Resolution 466/2012) and was submitted to the Research Ethics Committee (CEP) of the ACCG, having ACCG-Hospital Araujo Jorge as the proposing institution. The number of the assent, given in the embodied opinion of the CEP, was 4.235.164.

As the study was carried out by analyzing the forms of dispensing and use of antimicrobials, retrospectively, it was not necessary to apply the Informed Consent Form (FICF) for the participants involved. Patient data were managed and analyzed so as not to compromise their identity or integrity in any part of the process. 
Inclusion Criteria: antifungal application forms for patients under 19 years of age, admitted to the HAJ wards and ICU between January 1, 2016 and December 31, 2019, who used at least one antifungal agent (nystatin, fluconazole, itraconazole, voriconazole, echinocandin or amphotericin) for a period of hospitalization between January 1, 2016 and December 31, 2019.

Exclusion Criteria: patients aged 19 years or older; records of patients who did not present the patient's age, date of admission and used of antifungal agents; records whose patients had not used at least one antifungal agent during their hospital stay; patients who do not have a diagnosis of cancer as their underlying disease.

The present study is a retrospective cohort that analyzed all requests $(n=1211)$ of antifungal agents for pediatric cancer patients hospitalized between January 2016 and December 2019 in an oncology reference unit in the state of Goias.

Study location: since 1994, the Association for Combating Cancer of Goias (ACCG) has taken over the coordination and administration of the Population-Based Cancer Registry (RCBP) in Goias, integrating INCA's information network. ACCG is a private, philanthropic institution, certified by the Ministry of Health $(\mathrm{MH})$ as a Beneficent Entity for Social Assistance in Health, declared a Municipal Public Utility Entity; is affiliated with the Brazilian Association of Philanthropic Institutions to Fight Cancer (ABJFJCC). In Goias, Hospital Araujo Jorge, administered by ACCG, is the only High Complexity Care Center in Oncology by the Ministry of Health, and the only one in the Midwest Region authorized with the Pediatric Oncology and Bone Marrow Transplant Service. The Pediatric Oncology service at Hospital Araujo Jorge performed 38.458 outpatient care and 7.261 admissions (2.659 patients) in the last 6 years (2014 to 2019), and of the patients seen during this period, $92.63 \%$ came form Goiania and other cities in Goias; and $7.37 \%$ from other 14 states of the Federation. $^{9}$

Data collection: since the release for the use of any antimicrobial in the institution is subject to the completion of a digital request (electronic form) generated at the time of medical prescription, containing the epidemiological data related to the patients, underlying disease and justification of the antifungal prescribed, for evaluation by the SCIH (hospital infection control service) auditor physician; this information was accessed via the available 
institution information system (Omni and Tasy). Complementary data to the forms were accessed in the electronic medical record of the same information system.

Variables analyzed: age group, gender, underlying oncological disease, type of treatment for fungal infection, diagnostic criteria used for fungal infections, suspected fungal infection, drug used for treatment, modification of the antifungal, evolution and outcome of the infection assumed fungal.

Data analysis: breaking down the topics, the type of treatment for fungal infection was distributed into the following categories: prophylaxis, empirical therapy, preemptive therapy or targeted therapy. Regarding the diagnostic methods used, we have the following categorization: clinical-epidemiological, imaging, culture and microscopy studies, and the detection of the fungus components. Treatment behavior was stratified into: remission of the condition or lack of response. As for the addition or replacement of the prescribed antifungal, we have: yes or no; as to the reason for the modification, we have: not modified, new diagnosis of fungal infection, treatment intolerance, new fungal infection, retreatment. Cases of prophylactic use, without clinical fungal infection, were categorized with the topic: not applicable.

The data obtained through the forms were tabulated using the Excel® program according to the aforementioned categories. In which parameter we had the distribution of categories, where the frequency of each category was computed, which will be broken down in the results of this study in the form of percentages. Quantitative variables will be described through distribution by mean, median and standard deviation. The significance level used in the study was $\leq 0,05$ and a $95 \%$ confidence interval. Compliance with ethical principles is highlighted in this work, with the attachment of the CEP opinion at the end.

\section{RESULTS}

During the period from 2016 to 2019, 310 children were treated at the hospital in multiple admissions and used antifungal agents (1.211 times). Of these, 129 children (41.6\%) aged between 5 completed years to 10 incomplete years, followed by 97 children $(31.3 \%)$ aged between 10 completed years to 15 incomplete years, 65 patients $(21.0 \%)$ between 15 completed years to 19 incomplete years and 19 children (6.1\%) between 1 completed year to 5 incomplete years. Children aged between 0 months and 1 incomplete year did not use antifungal drugs in the period in question. 
Graph 1 - Distribution by age group

Distribution by age group

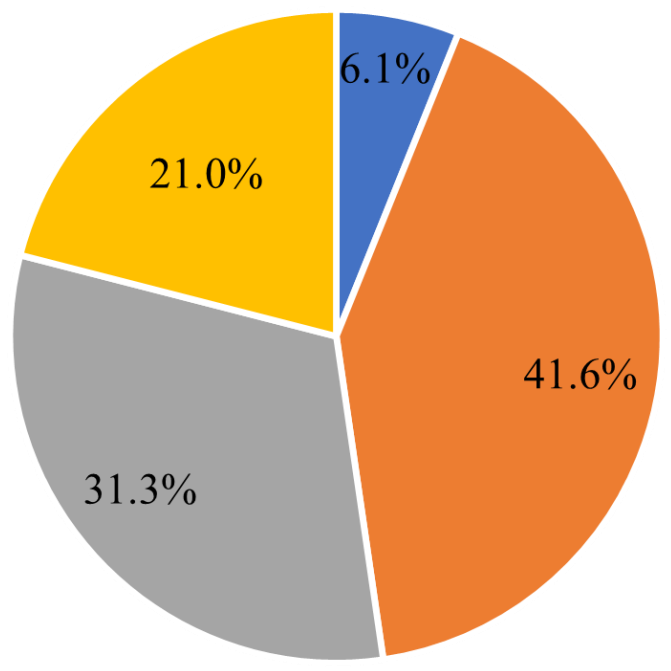

$-1<5$ years old

$\square<10$ years old

- $10<15$ years old

$\square 15<19$ years old

Source: prepared by the authors (2021)

Of the total of 310 hospitalized patients in the period, 163 children $(52.6 \%)$ were male, while 147 (47.4\%) were female. Regarding the underlying oncological disease, 158 children (51.0\%) were diagnosed with leukemia (135 ALL and 23 AML); 72 children (23.2\%) with central nervous system (CNS) tumors; 52 children (16.8\%) with other solid tumors; 26 children $(8.4 \%)$ with lymphomas and 2 children $(0.6 \%)$ had bone marrow aplasia (BMA).

Among the infections treat in 1.211 antifungal requests, mucocutaneous candidiasis, with or without mucositis, accounted for 776 cases (64.1\%); probable fungal pneumonia, 46 cases (3.8\%); invasive candidiasis, 44 cases (3.6\%) and other mycoses, 7 cases $(0.6 \%)$. As for the treatment of invasive aspergillosis, there were 25 cases $(2.1 \%)$ of specific therapy with a diagnosis based on positive galactomannan and/or culture. The remaining patients were asymptomatic from the point of view of fungal infection, considering that 46 (3.8\%) used secondary prophylaxis for invasive aspergillosis (previous diagnosis) and another 267 cases $(22 \%)$ used primary prophylaxis for candidiasis.

Regarding the use of antifungals, empirical treatment accounted for 870 cases (71.9\%), followed by prophylactic use in 313 cases $(25.8 \%)$ and only 28 cases $(2.3 \%)$ of 
antifungal use as directed therapy. No antifungal agents were requested as preemptive therapy.

As for the diagnostic methods justified in antifungal requests, the clinical and epidemiological criteria were 1.140 cases (93.3\%). Imaging studies (computed tomography) were 46 cases $(3.8 \%)$, detection of fungus components (galactomannan) was found in 25 cases $(2 \%)$ and culture for fungi and microscopy in only 11 cases $(0.9 \%)$. In total, 1.222 diagnostic resources were used for 1.211 cases of antifungal therapy; thus, it is observed that, in some children, more than one diagnostic method was used.

When evaluating the type of antifungal requested for use, nystatin was used in 588 cases $(48.6 \%)$; fluconazole accounted for 464 cases (38.3\%); amphotericin deoxycholate was requested in 86 cases $(7.1 \%)$; itraconazole, in 46 cases $(3.8 \%)$; voriconazole, in 26 cases (2.1\%); and only 01 case $(0.1 \%)$ used anidulafungin (one echinocandin).

Graph 2 - Distribution of antifungal treatments

Distribution of antifungals used

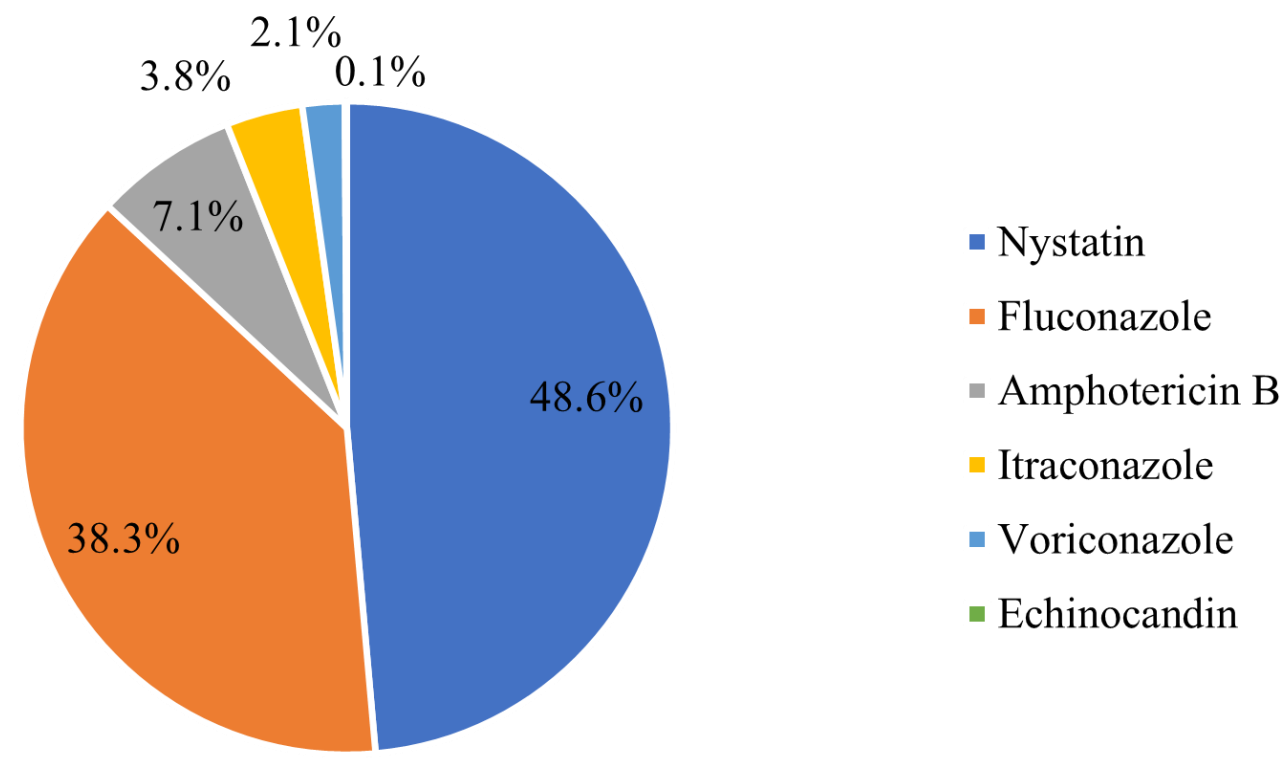

Source: prepared by the authors (2021)

From the cases assumed infections that received antifungal therapy, 718 (59.3\%) progressed to remission of the condition without description of change to the association of antifungal and 175 cases (14.5\%) did not respond to therapy (therapeutic failure). 
Of the 175 cases evaluated as therapeutic failure, in 174 cases, substitution or the addition of another antifungal agent was performed. Of the justifications for modifying therapy (174 cases), retreatment of fungal infection accounted for 82 cases $(6.8 \%)$, followed by 68 cases $(5.6 \%)$ of refractory fungal disease, and 65 cases $(5.4 \%)$ of an assumed new diagnosis. Drug intolerance occurred in two cases $(0.2 \%)$.

Graph 3 - Reason for changing/adding antifungal

Reason for exchanging/addition of antifungal

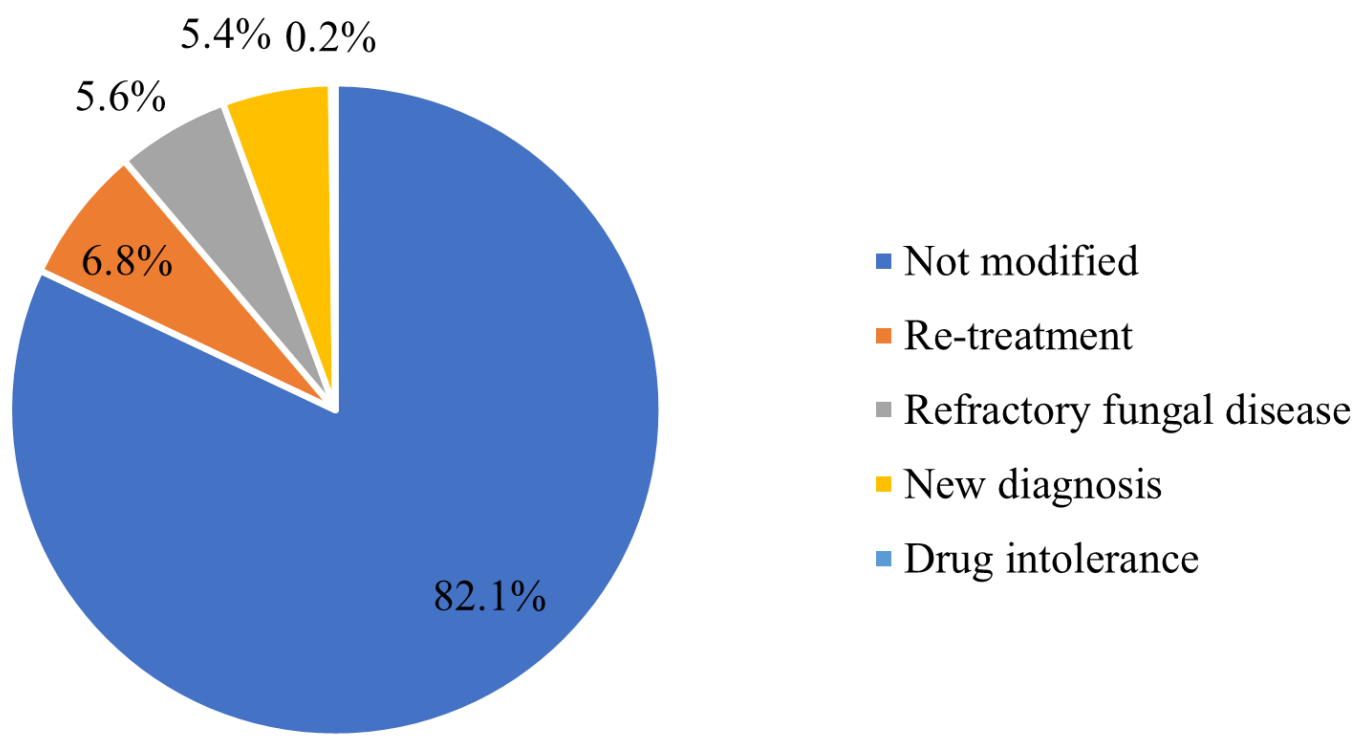

Source: prepared by the authors (2021)

During the study period, there were 114 episodes of invasive fungal infection (IFI), being: 32 cases of invasive candidiasis/sepsis (28.1\%); 11 cases of fungal esophagitis/colitis (9.6\%); 46 probable fungal pneumonias (40.4\%) and 25 cases of invasive aspergillosis $(21.9 \%)$. The IFIs were assumed by exclusive clinical-epidemiological criteria 40 times $(35.1 \%)$, by clinical-epidemiological criteria and suggestive image 46 times $(40.3 \%)$, by positive fungal cultures (yeasts in automated blood culture) in 3 times (2.6\%) and by positive serum galactomannan (cut off $\geq 500$ ) in 25 times $(22.0 \%$ ). 
Graph 4 - Distribution of invasive fungal infections

Distribution of invasive fungal infections

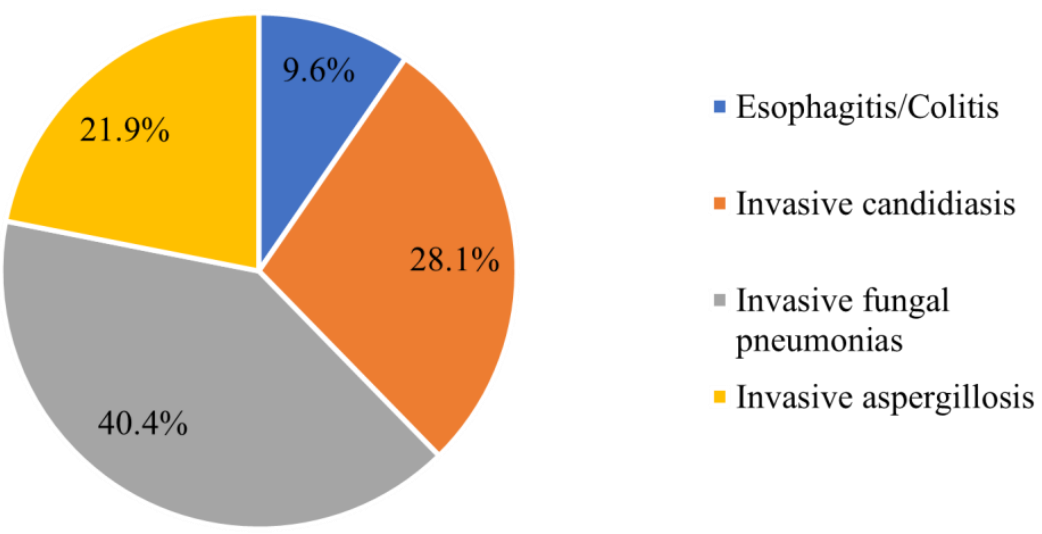

Source: prepared by the authors (2021)

For the treatment of IFIs, amphotericin B deoxycholate was used 81 times (71.0\%); voriconazole, 26 times (22.8\%); fluconazole was used 06 times (5.3\%) and anidulafungin only once $(0.9 \%)$.

\section{Graph 5 - Antifungal used in IFIs}

Antifungals used in IFIs

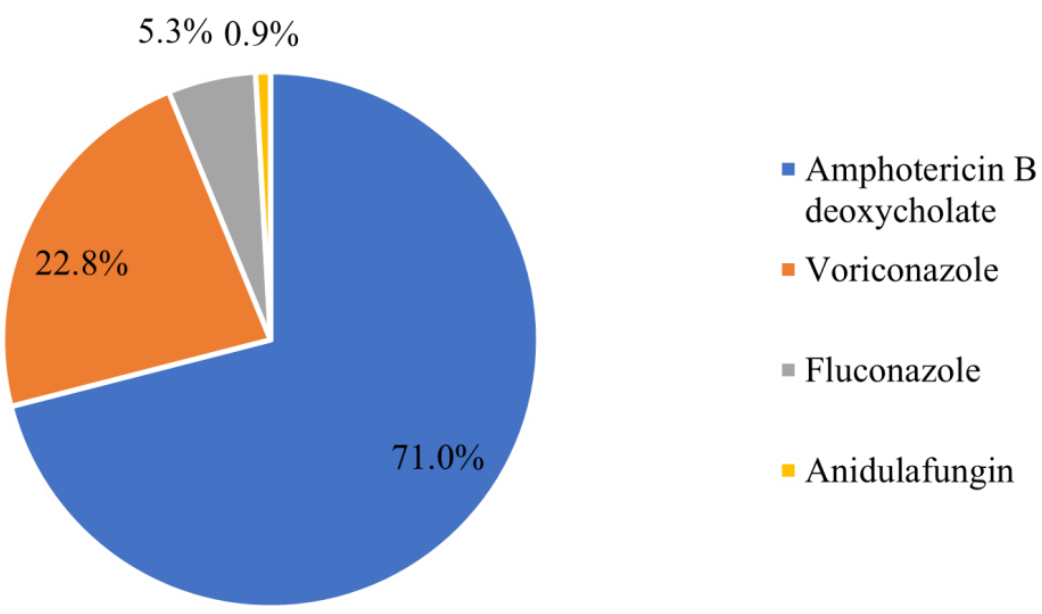

Source: prepared by the authors (2021)

Of the assumed invasive fungal infections, 82 progressed to fungal remission (71.9\%) and 32 cases $(28.1 \%)$ were considered refractory fungal disease. Among the cases 
refractory (32) to the use of antifungal agents, there were 18 deaths $(56.2 \%)$. There were also 8 deaths from other causes, among the cases of IFIs.

\section{DISCUSSION}

This study evaluated the epidemiology of fungal infections in the pediatric service of a reference hospital for the treatment of cancer in the state of Goias, from 2016 to 2019, by collecting data from the antifungal drug dispensing form in the hospital pharmacy. From 2016 to 2019 , the study hospital accounted for 4.830 pediatric admission, with an annual average of 1.207 admission. Considering the 1.211 requests for antifungal agents in the same period, as one request for each hospitalization episode, the use of at least one antifungal agent is expected for one in four pediatric hospitalizations. During this same period, there were 169 pediatric deaths in the study hospital, with an annual average of 42.2 deaths/yars. Thus, it is believed that four children die annually in the institution (10.65\% of annual deaths) as a result of an invasive fungal infection.

Regarding the population studied, of the 310 children who used some antifungal agent during hospitalization, $163(52.6 \%)$ were male, with a prevalent age group ranging from 5 complete years to 10 incomplete years (129 children - 41.6\%), and the underlying oncological disease in 158 children $(51.0 \%)$ was leukemia, mainly acute lymphoid leukemia (ALL) in 135 cases (85.4\%). These characteristic are similar to the epidemiological profile of the disease in the pediatric population, as there is a higher incidence of ALL compared to other types of neoplasms. ${ }^{10}$

Among the types of antifungal treatments used, empirical therapies were the majority $(71.9 \%)$, followed by prophylaxis $(25.8 \%)$. Directed treatments, that is, those in which fungus components are detected or in which there is a positive culture for fungi, accounted for only $2.3 \%$ of cases. Castagnola et all. ${ }^{11}$ (2006), in a study carried out in pediatric onco-hematological treatments centers in Italy, found that most cases of fungal infections were reported as proven and received targeted therapy. ${ }^{11}$ In a brazilian study, Brandi (2019) observed that a minority of patients had proven fungal infection (36\%) and received targeted therapy, similarly to the data found in our study $-2.3 \%{ }^{8}$

The most recent guidelines recommend, as preferred diagnostic procedures (gold standard), blood culture and microscopy for fungi, in addition to radiological imaging studies $^{12}$ and other known methods for detecting fungus components, such as serum 
galactomannan, (1,3)- $\beta$-D-glucan and the polymerase chain reaction - PCR, considered complementary. ${ }^{13}$

In the study hospital, the ELISA for galactomannan, the only test available for detecting fungus components, has been carried out at the institution since 2015, with an average of 463 tests per year in the period from 2016 to 2019. However, this diagnostic method, together with the culture and microscopy for fungi, occurred in only $2.9 \%$ of cases, pointing to the need to optimize the indication and diagnostic resources performed in pediatrics.

Of the fungal infections treated during the study period, mucocutaneous candidiasis, associated or not with mucositis, accounted for the majority of diagnostic justifications in antifungal requests (64.1\%). Probable fungal pneumonia was the second highest justification (3.8\%), followed by invasive candidiasis (3.6\%) and invasive aspergillosis $(2.1 \%)$.

Infections associated with the genus Candida spp. in the study, they account for $64.7 \%$ of the cases. Similar to these results, other studies highlight Candida spp. as the most frequent pathogenic fungi in humans and indicate that mucositis is a very common side effect of chemotherapy treatment in children, which may or may not be associated with moniliasis. ${ }^{4,14}$ This explains why, despite the availability of some diagnostic methods in the hospital, most fungal infections were treated and prevented by clinical and epidemiological criteria (93.3\%), contrary to the recommendations of the guidelines.

In primary prophylactic therapy with antifungals, the guidelines recommend the use of the following drugs: fluconazole, micafungin and liposomal amphotericin B. ${ }^{12}$ Our study found the frequent use of nystatin $(48.6 \%)$ as primary antifungal prophylaxis in leukosis, a practice of low evidence scientific and not recommended. Studies show that, with regard to colonization by fungi, fluconazole did not demonstrate superiority over nystatin; however, with regard to invasive fungal infection, fluconazole showed better results, and is therefore the drug of first choice in antifungal prophylaxis. ${ }^{15}$

With regard to mucocutaneous candidiasis, the reference hospital in this study has this infection as the first position in the diagnosis of fungal infections (64.1\%). In most cases, it appears, also, that the most used drugs are those related to the prophylaxis and/or treatment of mucositis, being, therefore, nystatin in first place with 588 prescriptions and fluconazol in 
second place with 464 prescriptions in the period. These data are in line with the proposed literature, evidencing here the study by Flynn et al.16 (1999) in which these two drugs are the most used for this pathology. ${ }^{16}$

However, contrary to the prescriptions being in agreement with the proposed literature, the first treatment option does not coincide. In the study by Flynn et al., we have a remarkable superiority of fluconazole over nystatin (almost double) in the clinical cure rate. Knowing this superiority in treatment, the preferential and, quite significant, use of nystatin in the context of the reference hospital in Goias for the management of cases of mucocutaneous candidiasis in immunosuppressed pediatric patients due to cancer history is questioned. ${ }^{16}$

Regarding the use of empirical therapy, our study found amphotericin B deoxycholate $(\mathrm{ABD})$ as the most used antifungal. Studies indicate that ABD almost always results in some degree of renal dysfunction due to its nephrotoxicity. ${ }^{17}$ Taking this into account, the use of liposomal amphotericin B is recommended. ${ }^{15}$

Invasive fungal infections, of greater clinical and epidemiological importance, occurred in a total of 114 episodes $(9.4 \%)$ of all cases analyzed in this study. Considering all hospitalizations of children in the four years studises - 4.830 admissions, we have an even lower percentage: $2.3 \%$.

In terms of the type of infection, they were represented in four groups: probable fungal pneumonia with $40.4 \%$ (46 cases), followed by invasive candidiasis associated with sepsis with $28.1 \%$ (32 cases) and esophagitis/colitis with $9.6 \%$ (11 cases); and invasive aspergillosis with $21.9 \%$ (25 cases).

When analyzing the diagnostic criteria of the IFIs, again, it is noticed the predominance of purely clinical-epidemiological criteria in $35.1 \%$, or even associated with suggestive diagnosis by radiological imagem $(40.3 \%)$ as in the total cases studied. The positive result of galactomannan was reported in $22 \%$ of the cases, a percentage considered low compared to clinical and imaging criteria, as it is an effective diagnostic method available in the service. Fungal culture was used in only 3 cases, $2.6 \%$, being the least used method to confirm the diagnosis of IFIs. Knowing that the use of imaging to complete the diagnosis was the most used method in the study hospital, we have a contradiction regarding the most current guideline in which Groll et al. ${ }^{12}$ shows that imaging findings are generally nonspecific for fungus and that, particularly in children under 5 years of age, the typical signs of 
fungal pneumonia may not be found, with signs similar to nodules of soft tissue masses being more common. $^{12}$

Regarding the treatment of the invasive infections, amphotericin B deoxycholate was the drug most used by the mentioned service, occurring in $71 \%$ of the prescriptions. Next to it are: voriconazole, fluconazole and anidulafungin. In a study by Cohen-Wolkowiez et $a l .{ }^{15}$ it was noted that the use of amphotericin B deoxycholate has decreased due to the emergence of antifungal agents with better safety profiles. Although amphotericin deoxycholate is better tolerated in children than in adults and comparative studies with its lipid formulations (complex lipid or liposomal amphotericin) do not change mortality, guidelines recommend the use of lipid amphotericin for neutropenic children in chemotherapy treatment. $^{15}$

Blyth et al. ${ }^{18}$ (2007) showed that despite constant improvements in supportive care, the mortality rate from IFIs is still considered high and quite costly to health systems. In congruence with the study by Blyth, a similarity with this high mortality rate $(56.2 \%)$ was found in the HAJ for children with IFIs refractory to antifungal therapy, with 18 deaths in 32 recurrences. $^{18}$

In the same period of 2016 to 2019, there were 169 pediatric deaths in the study hospital, with an annual average of 42.2 deaths/year. Thus, it is believed that four children die annually in the institution (10.65\%) of annual deaths) as a result of an invasive fungal infection.

\section{CONCLUSION}

Fungal infections assumed in the pediatric service of the cancer hospital studied are epidemiologically associated with cases of onco-hematological disease (leukemia), primarily by clinical and epidemiological criteria, through the prophylactic and empirical use of low-spectrum antifungal agents (fluconazole and nystatin). The use of empirical therapies prevails, which are not appropriate to define the indicated antifungal drugs, adequate period for use, and the need for retreatment or therapeutic association.

The diagnostic resources available at the unit for invasive fungal infections are scarce and focus on radiological imaging exams and dosage of galactomannan, considered complementary exams, which do not support a targeted and effective therapy. 
There is a need for adjustments in the recommendations of antifungal prophylaxis, especially in the use of nystatin, and empirical therapy with amphotericin deoxycholate and its complications, according to the most recent guidelines, with regard to target patients for this type of treatment. Preferably, ensure the use of safer and more effective therapeutic options such as echinocandins in invasive candidiasis, voriconazole in invasive aspergillosis and lipid formulations of amphotericin in other systemic mycoses.

Finally, finding high mortality rates from IFIs both in the study hospital and in the literature, we suggest coordinated actions to optimize the therapeutic and diagnostic resources of IFIs in high-risk patients, to reduce hospital costs and improve survival and care palliatives.

\section{REFERÊNCIAS}

1. INSTITUTO NACIONAL DE CÂNCER JOSÉ ALENCAR GOMES DA SILVA (INCA). Estimativa 2020: incidência de câncer no Brasil. Rio de Janeiro: 2019. Disponível em: <https://www.inca.gov.br/sites/ufu.sti.inca.local/files/media/document/estimativa2020-incidencia-de-cancer-no-brasil.pdf>. Acesso em: 09 Mar; 2020.

2. MINISTÉRIO DA SAÚDE. Protocolo de Diagnóstico Precoce do Câncer Pediátrico. Brasília, 2017. Disponível em: <http://bvsms.saude.gov.br/bvs/publicacoes/ protocolo_diagnostico_precoce_cancer_pediatrico.pdf>. Acesso em: 09 Mar. 2020.

3. RAMOS, J. T. et al. Clinical practice update of antifungal prophylaxis in immunocompromised children. Revista Espanola de Quimioterapia, Madri, v. 32, n. 5, p. 410-425, 2019.

4. FRACCHIOLLA, N. S. et al. Epidemiology and treatment approaches in management of invasive fungal infections in hematological malignancies: Results from a single-centre study. PLoS ONE, São Francisco. 14, n. 5, p. 1-14, 2019.

5. LAMOTH, F. et al. Changing epidemiology of invasive mold infections in patients receiving azole prophilaxis. Clin Infect Dis, Oxford 2017.

6. DE PAUW, B. et al. Revised Definitions of Invasive Fungal Disease from the European Organization for Research and Treatment of Cancer/Invasive. Clin. Infect. Dis, Oxford, v. 46, n. 12, p. 1813-1821, 2008.

7. NIEDERHUBER, J. E. et al. Abeloff's clinical oncology. 5 ed. Filadélfia: Elsevier, 2014. 
8. BRANDI, A. C. M. B. Infecções fúngicas invasivas em crianças e adolescentes com câncer. 2019. 119p. Dissertação (Mestrado em Ciências) - Faculdade de Medicina, USP, São Paulo, 2019.

9. ASSOCIAÇÃO DE COMBATE AO CÂNCER EM GOIÁS (ACCG). Demonstrativo de Atividades de 2018. 2020. Relatório Anual da ACCG - ACCG, Goiânia.

10. SANTANA, L. R. et al. Perfil epidemiológico das Leucemias em Crianças e Adolescentes no Estado da Bahia. Gaz. méd. Bahia, Salvador, v. 77, n. 1, p.51-54, 2007.

11. CASTAGNOLA, E. et al. Fungal Infections in Children With Cancer: A Prospective, Multicenter Surveillance Study. Pediatr Infect Dis J, Filadélfia, v. 25, p. 634-639, 2006.

12. GROLL, A. H. et al. Fourth European Conference on Infections in Leukaemia (ECIL-4): guidelines for diagnosis, prevention, and treatment of invasive fungal diseases in paediatric patients with cancer or allogeneic haemopoietic stem-cell transplantation. Lancet Oncol, Londres, v. 15, p. e327-40, 2014.

13. LEHRNBECHER, T. et al. Guideline for the Management of Fever and Neutropenia in Children With Cancer and Hematopoietic Stem-Cell Transplantation Recipients: 2017 Update. J Clin Oncol, Alexandria, v. 35, p.2082-2094, 2017.

14. MENEZES, A. C. et al. Abordagem clínica e terapêutica da mucosite oral induzida por radioterapia e quimioterapia em pacientes com câncer. Rev Bras Odontol, Rio de Janeiro, v. 71, n. 1, p.35-38, 2014.

15. COHEN-WOLKOWIEZ, M. et al. Pediatric antifungal agents. Curr Opin Infect Dis, Londres, v. 22, p. 553-558, 2009.

16. FLYNN, P. M. et al. Oropharyngeal candidiasis in immunocompromised children: a randomized, multicenter study of orally administered fluconazole suspesion versus nystatin. The Multicenter Fluconazole Study Group. J Pediatr, v. 127, n. 2, p. 322328.

17. FILIPPIN, F. B., SOUZA, L. C. Eficiência terapêutica das formulações lipídicas de anfotericina B. Revista Brasileira de Ciências Farmacêuticas, São Paulo, v. 42, n. 2, p. 167-194, 2006.

18. BLYTH, C. C., PALASANTHIRAN P., O'BRIEN T. A. Antifungal Therapy in Children With Invasive Fungal Infections: A Systematic Review. PEDIATRICS, v. 119, n. 4, p. 772-784, 2007. 